\title{
Offline Signature Verification in Punjabi based on SURF Features and Critical Point Matching using HMM
}

\author{
Rajpal Kaur
}

\begin{abstract}
Biometrics, which refers to identifying an individual based on his or her physiological or behavioral characteristics, has the capabilities to the reliably distinguish between an authorized person and an imposter. The Signature recognition systems can categorized as offline (static) and online (dynamic). This paper presents Surf Feature based recognition of offline signatures system that is trained with low-resolution scanned signature images. The signature of a person is an important biometric attribute of a human being which can be used to authenticate human identity. However the signatures of human can be handled as an image and recognized using computer vision and HMM techniques. With modern computers, there is need to develop fast algorithms for signature recognition. There are multiple techniques are defined to signature recognition with a lot of scope of research. In this paper, (static signature) off-line signature recognition \& verification using surf feature with HMM is proposed, where the signature is captured and presented to the user in an image format. Signatures are verified depended on parameters extracted from the signature using various image processing techniques. The Off-line Signature Verification and Recognition is implemented using Mat lab platform. This work has been analyzed or tested and found suitable for its purpose or result. The proposed method performs better than the other recently proposed methods.
\end{abstract}

\section{Keywords}

Offline Signature verification, offline signature recognition, signatures, SURF features and HMM.

\section{INTRODUCTION}

In our society, traditional and accepted means for a person to identify and authenticate himself either to another human being or to a computer system is based on one or more of these three general principles:

- What the person knows

- What he possesses or

- What he is

The written signature is regarded as the primary means of identifying the signer of a written document based on the implicit assumption that a person's normal signature changes slowly and is very difficult to erase, alter or forge without detection. The handwritten signatures are one of the different ways to authorize transactions and authenticate the human identity compared with other electronic identification methods such as fingerprints scanning and retinal vascular pattern screening. The signature verification is categorized into two parts that is static or offline signature and dynamic or online signatures. In offline signature verification, data acquisition done by scanning the image and in data acquisition done by stylus, digitizer, touch screen. In this paper we are dealing with an offline or static signature verification technique. The Offline signatures are more challenging than online because the features are extracted from the static 2-D image of signatures. The main difficulties in offline signatures are

\author{
Pooja Choudhary
}

unconventional writing and highly stylish signatures, the nonrepetitive nature of variation of signatures because of age, illness, stress level and geographical locations to some extent the emotional state of the person. Offline signature verification is considered inevitable to determine if a particular signatures is valid i.e. genuine or forged. There are three main types of forgeries as: Random Forgery, Simple Forgery, and skilled Forgery. In the Random Forgery, the forger does not know the signature shape and name of signer. In unskilled or simple forgery, the forger knows the signer name but not the shape of the signatures. In skilled forgery, the forger has experience in copying the signature and genuine signature produced by forger.

\section{PROBLEM FORMULATION}

The problem of handwritten signature verification is to Verifies whether the test signatures are original or forged. The offline signature verification is the important part of the biometrics. The biometrics is act of science to verify and identify a human being identity through Behavioral and physiological measurements. Offline signature verification and identification can be done by behavioral biometric system include (keystrokes, gait and gestures, voiceprint). The signatures depends on few factors like physical, psychological state of the person, body position, writing surface or writing material (pen), purpose of signing, environmental factors. The behavioral characters can change over time, but it less expensive and less dangerous to users and not Easley transferable of every person, cannot lost and broken. The choice one of the biometric solutions depends on several factors which include:

- User acceptance

- Level of security required

- Accuracy

- Cost and implementation time

The method of signature verification reviewed in this paper benefits the advantage of being highly accepted by potential customers. The use of the signature recognition has a long history which goes back to the appearance of writing itself [9]. Utilization of the signature as an authentication method has already become a tradition in the western civilization and is respected among the others. The signature is accepted proof of person identity of the person in a transaction taken on his or her behalf. Then the users are likely to approve this kind of computerized authentication method. Signature verification systems differ in both their feature selection and their decision methodologies. There are more 40 different feature types have been used for signature verification.

\section{METHODOLOGY}

We approach the problem in two steps. Initially, scanned signature of image is preprocessed to be suitable for extracting features. After then, the preprocessed image is used to extract relevant geometric parameters that can distinguish forged signatures from exact ones using the HMM approach. 


\section{Preprocessing:}

The signature is first captured and transformed into a format that can be processed by a computer. The preprocessing is refers to the improvements in signature image after scanning. The preprocessing is applied on both training data and testing data set. Some preprocessing steps that are we have to use is discussed below:

1. Thinning: Thinning means reducing shapes of objects or eliminate the thickness. Thinning cleans the image and reduces the character features help in feature extraction and classification.

2. Noise Reduction: The handwritten scanned signature contains a lot of noise. Noise removed and restore captured image using noise filters. Noise removal is also called smoothing. The median filter is mostly used for smoothing.

3. Dilation of signatures image: It compensate small existing gaps along the signature resulting from the signature variability using dilation operations.

4. Rotation of Image: Rotation drops and reduces the common features between the training set and testing signature input. The correctness of the result depends on rotation algorithm orientation correction. Rotation of signature $e^{-t i \theta}$ is inevitable as time domain approach is precise to angle.

\section{Feature Extraction:}

The output from the preprocessing stage is given as input to the feature extraction stage. Feature extraction is the process of extracting the attributes of an image and accuracy of system also depends on it. The feature extraction for offline signature verification can be divided into three categories: Global features, Local features, Geometric features. In this paper we extract the geometric features that based on shapes and dimensions of signature image. The features that we employed are:

1. Area: Total number or actual number of Pixels in the regions.

2. Centriod coordinates: The Centriod coordinates are the vertical and horizontal centers of gravity of the signatures.

3. Eccentricity: The Eccentricity is the proportion of the distance between the foci of ellipse and length of its major axis.

4. Kurtosis: Kurtosis measures the flatness of distribution and gives the idea of whether the data is peaked or flat relative to natural or expected distribution.

5. Skewness: Skewness measures the asymmetry of distribution. A data set is symmetric if it looks the same to the left and right of the center point.

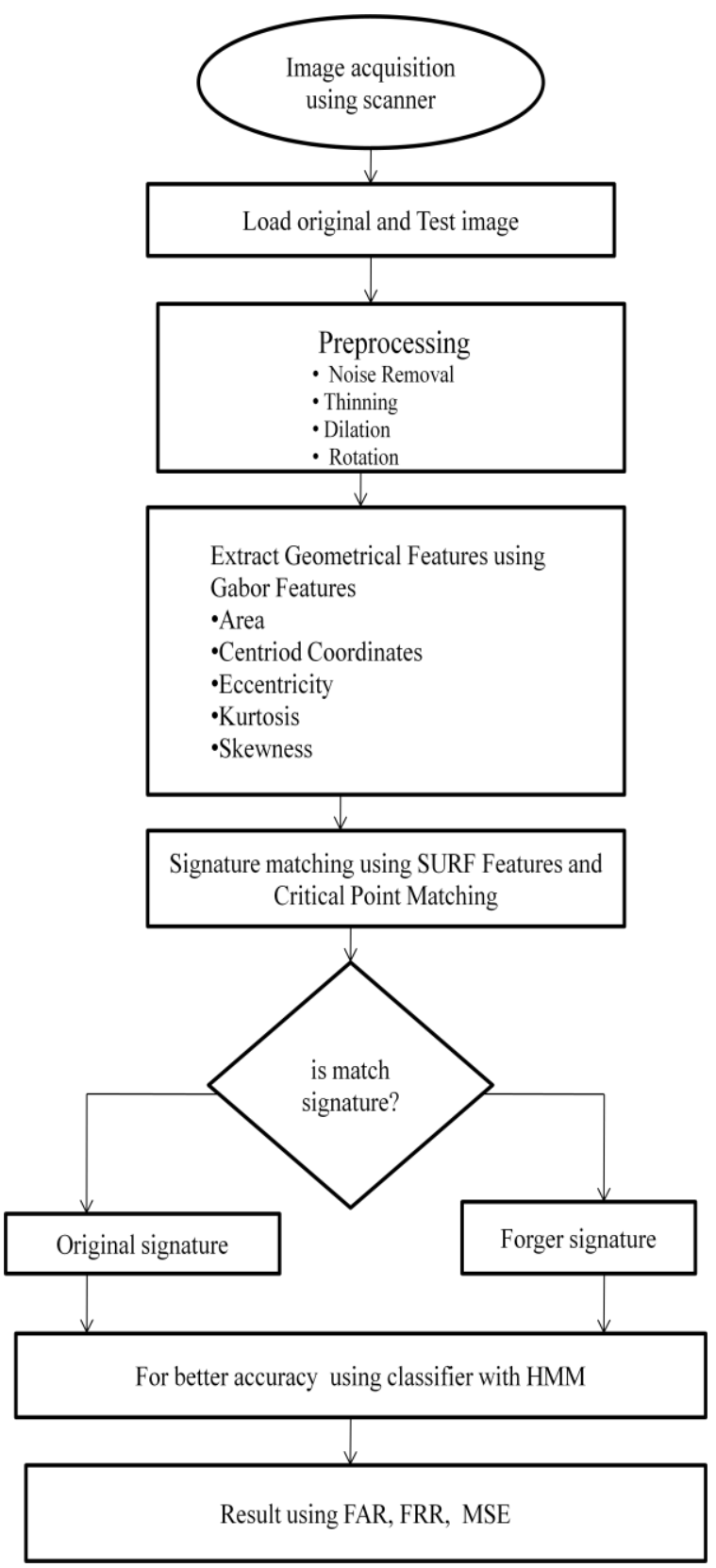

Figure 1: Block diagram of Approved work on signature verification

We use the Gabor filter for feature extraction. The Gabor filter is tunable band pass filter, multiscale, and multiresolution filter. Gabor filter have both spatial selective and frequency selective properties. The Gabor filter has the following general form in spatial domain:

$$
\begin{aligned}
\psi(x, y) & =\frac{f^{2}}{\pi \gamma \eta} e^{-\left(\frac{f^{2}}{\gamma^{2}} x^{\prime 2}+\frac{f^{2}}{\eta^{2}} y^{\prime 2}\right)} e^{j 2 \pi f x^{\prime}} \\
x^{\prime} & =x \cos \theta+y \sin \theta \\
y^{\prime} & =-x \sin \theta+y \cos \theta .
\end{aligned}
$$

In the equation $\mathrm{f}$ is the central frequency of the filter, $\theta$ the rotation angle, $\gamma$ sharpness along the Gaussian major axis, $\eta$ sharpness along the Gaussian minor axis. The features extracted at different rotation angles. 


\section{Surf features and Critical Point Matching:}

SURF: The SURF is faster and novel method for scale, affine, perspective and rotation invariants interest point detector and interest point descriptor. SURF is partially stirred and simulated by SIFT (scale invariant feature transform) descriptor. The computing speed of SURF is 3-5 times faster than SIFT. SURF is more resilient to noise than SIFT and more easily adaptive parallel processing than SIFT. The SURF has high repeatability means it has high reliability of discover same interest points under different viewing conditions. The SURF is progressively process in which firstly we find out the image interest points using determination of hessian matrix. After it find the interest point in scale space by non-maximal suppression on scale interest point maps. Then finding major interest points by finding the feature "direction" (works on the rotationally invariant features). The last and final step is to generate the feature vectors.

SURF is based on square matrix; the determinant of matrix is product of eigenvalues or sum of 2-D Haar wavelet reaction and formulates capable use of integral image. The interest points are chosen at typical or individual locations in the image, such as blobs, corners and T-junctions. The neighborhoods of each interest point are signifying a feature vector. The Descriptor has to be different and at same time robust to noise, geometric ad photometric deformations and detection displacements. as a result the descriptor vector are matches the two different images. The matching of features is based on distance between the vectors.

Critical point matching: To extract the critical points a contour based approach is used. In this approach the contour is traverse and any pointed or sharp change in the curve is marked as a critical point. The critical points can be best described as the set of points which form the basic structure of the signature. They are minimum set of points to represent the shape of signature.

\section{Hidden Markov Model (HMM):}

A Hidden Markov Model (HMM) Toolbox within the Mat lab environment is presented. The predictable methods in this toolbox, for the continuous and discrete HMM are developed for the training as well as for the test stage. The capability to create special group of components for the vector pattern is provided. The Multilabeling methods or techniques for the discrete HMM are also provided. The toolbox consist of procedures suitable for the classical applications defined on the HMM, as pattern Recognition, speech recognition and DNA sequence analysis. A HMM model is basically a stochastic finite state automaton, which provide an observation string, that is, the numbers or sequence of observation vectors, $\boldsymbol{O}=\boldsymbol{O} 1, \ldots, \boldsymbol{O} t, \ldots, \boldsymbol{O} T$. Thus, a HMM model consists of a number of N states $S=\{S i\}$ and of the observation string produced as a result of emitting a vector $\boldsymbol{O} t$ for each successive transitions from one state $S i$ to a state $S j$. $\boldsymbol{O} t$ is dimension and in the discrete case take it values in the library of $\mathrm{M}$ symbols. The probability of state transition and distribution between state $S i$ to $S j$ is $A=\{a i j\}$, and the observation probability distribution of emitting any Vector $\boldsymbol{O} \boldsymbol{t}$ at state $S j$ is given by $B=\{b j(\boldsymbol{O} t)\}$. The probability distribution of initial state is

$$
\begin{aligned}
& \Pi=\{\pi \mathrm{i}\} . \\
& a i j=(\text { ) } k \text { } ~ j k \text { i } P \text { q }=S q=S+(1) \\
& b j(\boldsymbol{O} t)=(\text { ) } t \text { t j P O } q=S(2) \\
& \pi i=(\text { ) } 0 \text { i P } q=S \text { (3) }
\end{aligned}
$$

Then, the given observation a HMM model $\lambda=(A, B, \Pi)$, and sequence $\boldsymbol{O}$, we can compute $P(\boldsymbol{O} \mid \lambda)$ the probability of the observed sequence by means of the forward-backward procedure [12]. Concisely, the forward variable is defined as the probability of the partial observation sequence $\boldsymbol{O} 1, \boldsymbol{O} 2 \ldots$ $\boldsymbol{O t}$ (until time $t$ ) and state Si at that time $t$, with the model $\lambda$, as $\alpha t(i)$. And the backward variable is defined as the probability of the partial observation sequence form $t+1$ to the end, given state $S i$ at that the model $\lambda$, as $\beta$ and time $t t(i)$. The probability of the observation sequence is calculated as:

$$
P(O \mid \lambda)=\sum_{i=1}^{N} \alpha_{t}(i) \beta t(i)=\sum_{i=1}^{N} \alpha_{T}(i)
$$

\section{ALGORITHM}

The SURF (speeded up robust features) is performance scale and rotation invariant interest point detection and descriptor. SURF algorithm is the feature point extraction algorithm. It is purposed by Bay H, Tuytellars T, and Gool L V in 2006. This algorithm is similar with SIFT algorithm. But, at the same time, it is three times faster than SIFT in calculate speed. SURF algorithm is composed of the following two parts.

\subsection{Feature Point Detection}

Significantly performance in SURF can be recognized to the use of intermediate image representation known as the Integral Image. The integral image is computed rapidly from an input image and is used to speed up the calculation Of any upright rectangular area. Integral image is defined by Jones M and Viola $\mathrm{P}$ and is represented by I L: (x). the value of point $\mathrm{X}=(\mathrm{x}, \mathrm{Y})$ is sum of pixels from the image origin to the $\mathrm{X}$ vertices.

$$
\begin{aligned}
& \mathrm{FLex})=\operatorname{LLf}(i, j) \\
& \mathrm{i}=\mathrm{O} \mathrm{j}=\mathrm{O}(1)
\end{aligned}
$$

The SURF detector is based on the determinant of the Hessian matrix. We can calculate the Hessian matrix based on Integral Image, as function of both space $\mathrm{x}=(\mathrm{x} ; \mathrm{y})$ and scale $\mathrm{a}$.

Here $\mathrm{L} x \mathrm{x}(\mathrm{x}, \mathrm{a})$ refers to the convolution of the second $\mathrm{d} 2 \mathrm{~g}$ (cr) order Gaussian derivative $\mathrm{dX} 2$ with the image at point $\mathrm{X}=$ (x, Y) and similarly for $\mathrm{L}$ yy and $\mathrm{L} x y$. If we use Weighted Box latter approximations in the $\mathrm{x}, \mathrm{y}$ and $\mathrm{xy}$ directions, the formula as an accurate approximation for the Hessian determinant using the approximated Gaussians

$$
\text { Det }(\text { Happrox })=\text { DxxDyy-(0.9Dxy) } 2
$$

As the processing time of the kernels used in SURF is size invariant, the scale-space can created by applied kernels of increasing size to the original image. That is allows for multiple layers of the scale-space pyramid to be processed simultaneously and negated the need to subsample the image hence providing performance increase.

The scale-space is divided into a number of octaves. In SURF, the lower level of the scale-space is obtained from the Output of the $9 \mathrm{x} 9$ filters shown in 2 levels. These filters correspond to a real valued Gaussian with $(\mathrm{J}=1.2$. As the Filter size increases so too does the value of the associated Gaussian scale and four layer making 1 Octave, we make the Parameter setting for 4 . With increasing the threshold, the number of detected interest points lowers and leave the only strongest one. After threshold is detected, a non-maximal suppression is performed to find a set of candidate points. In this process each pixel in the scale-space is compared to its 26 neighbors, 
comprised of the 8 points in the native scale and the 9 in each of the scales above and below.

\subsection{Interest Point Descriptor}

Interest point descriptor can be divided into two different tasks. First every interest point is assign a reproducible orientation before a scale dependent window is constructed in which a 64-dimensional vector is extracted. Second, defines the descriptor vector. To determine the orientation, Haarwavelet responses of size 4 ( $\mathrm{J}$ are calculate for a set pixels within a radius of 6 ( $\mathrm{J}$ of the detected point, the dominant orientation is $1 \mathrm{r}$ selected by rotating a circle segment covering an angle of 3 around the origin. At each position, the $\mathrm{x}$ and $\mathrm{y}-$ responses within the segment are summed and used to form a new vector. The large vector lends its orientation the interest point. Extracting the SURF descriptor need construct a square window around the interest point. This kind of window contains the pixels which will form entries in the descriptor vector and is of size 20 (J. This window is divided into $4 \mathrm{X} 4$ regular square sub regions. Then calculating the $\mathrm{d} \mathrm{x}$ and $\mathrm{d} Y$ of the sub region and weighting with a Gaussian $(3(\mathrm{~J})$, the descriptor means:

$\mathrm{v}=\left(\mathrm{L} d x, \mathrm{~L} d \mathrm{Y}^{\prime}\right.$ L Idx I, L Idyl) (4).

\section{EXPRIMENTAL RESULTS}

section presents the better results of the experiments to corroborate the success of the proposed model.

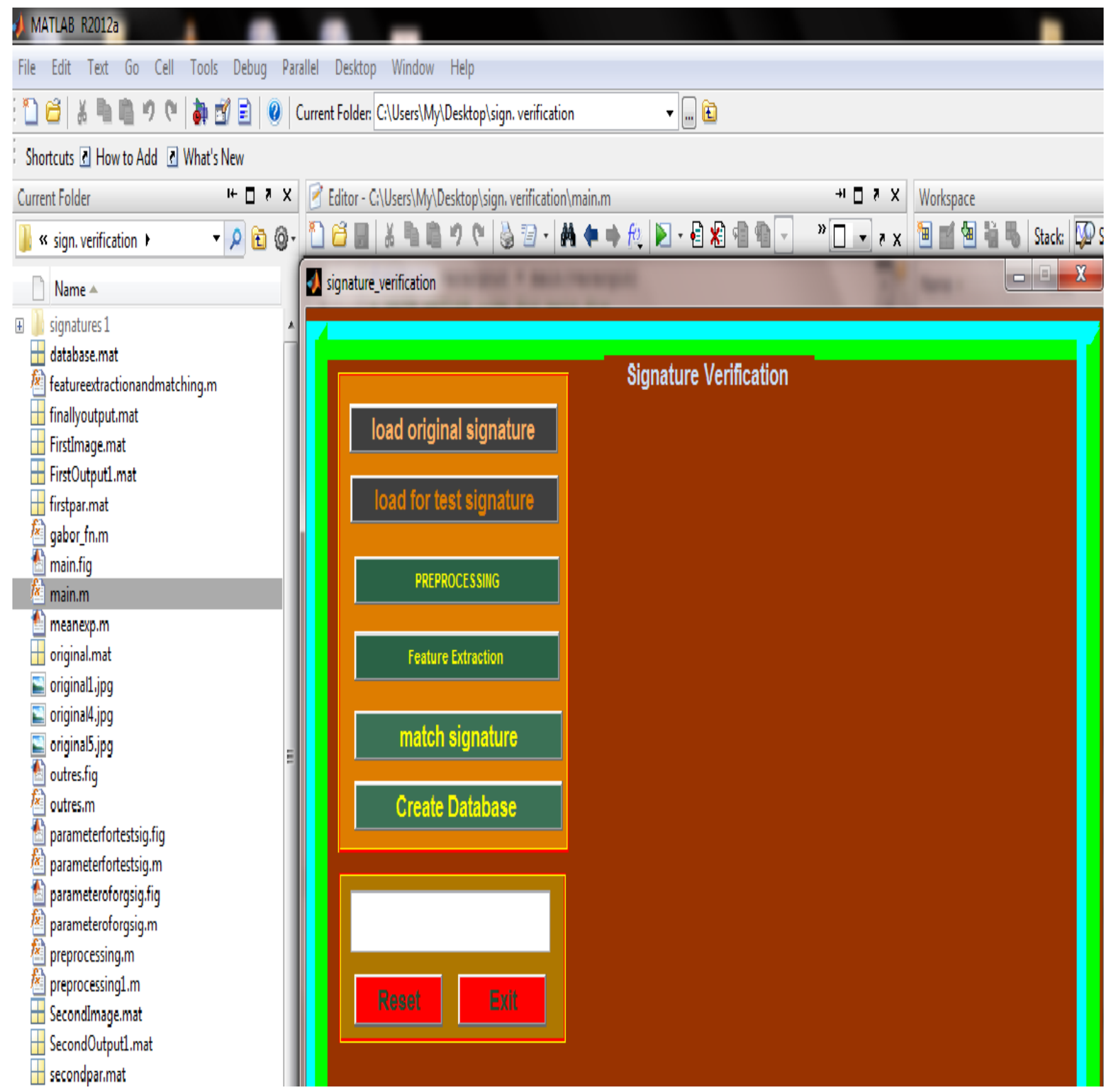

Figure 2: Signature verification process 
A MATLAB R2012a

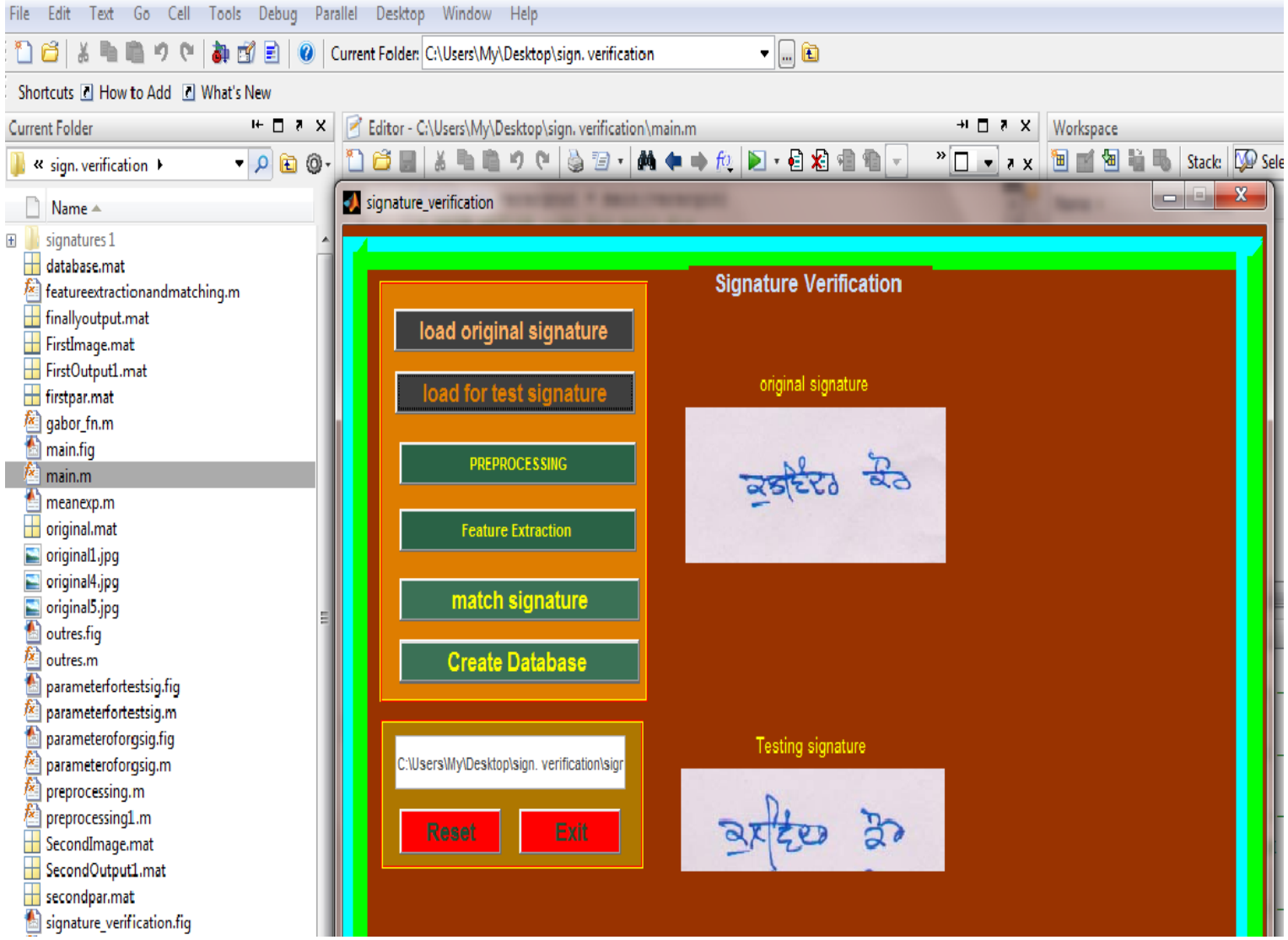

Figure 3: Load the original image and test image for signature verification

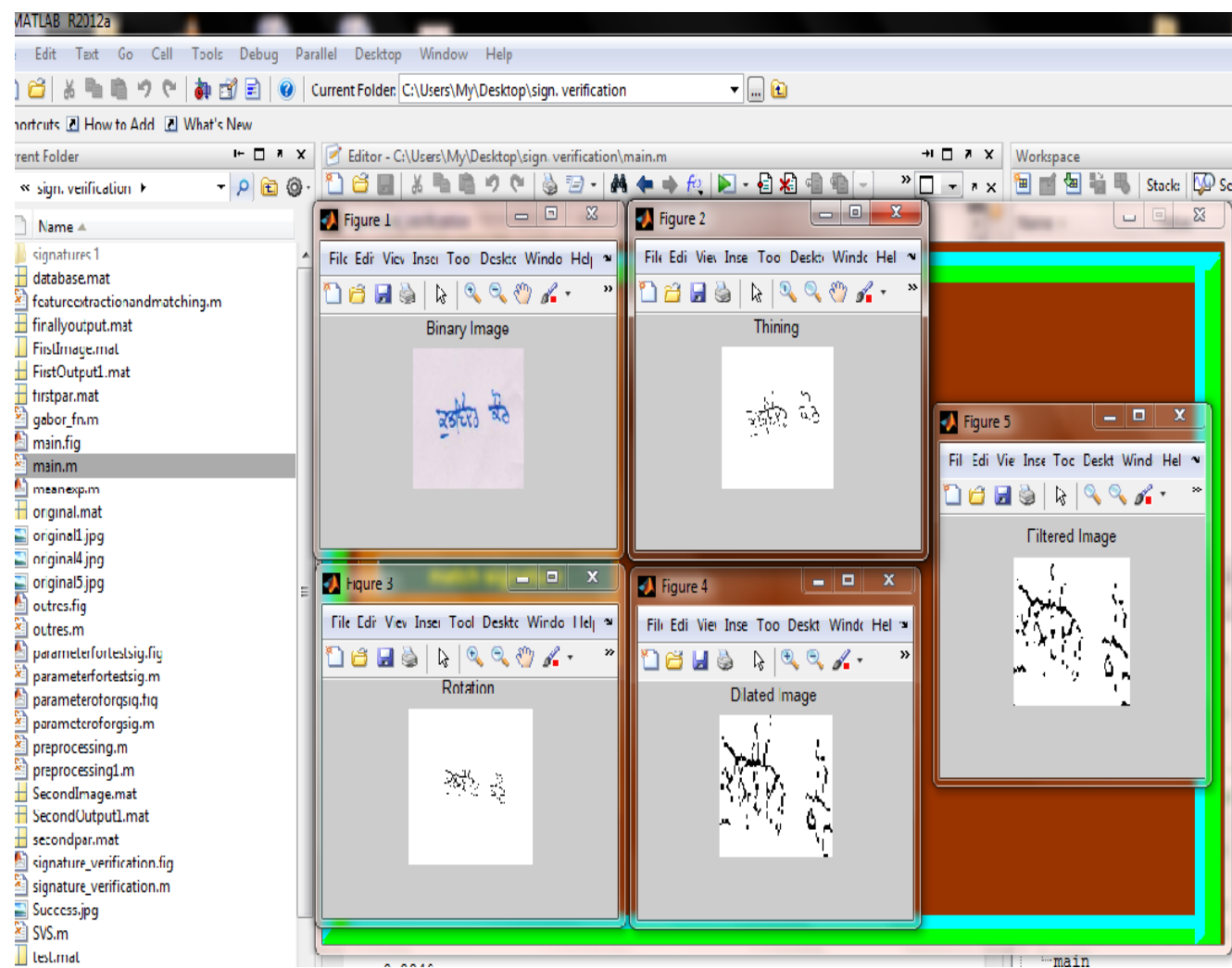

Figure 4: Preprocessing of original image 


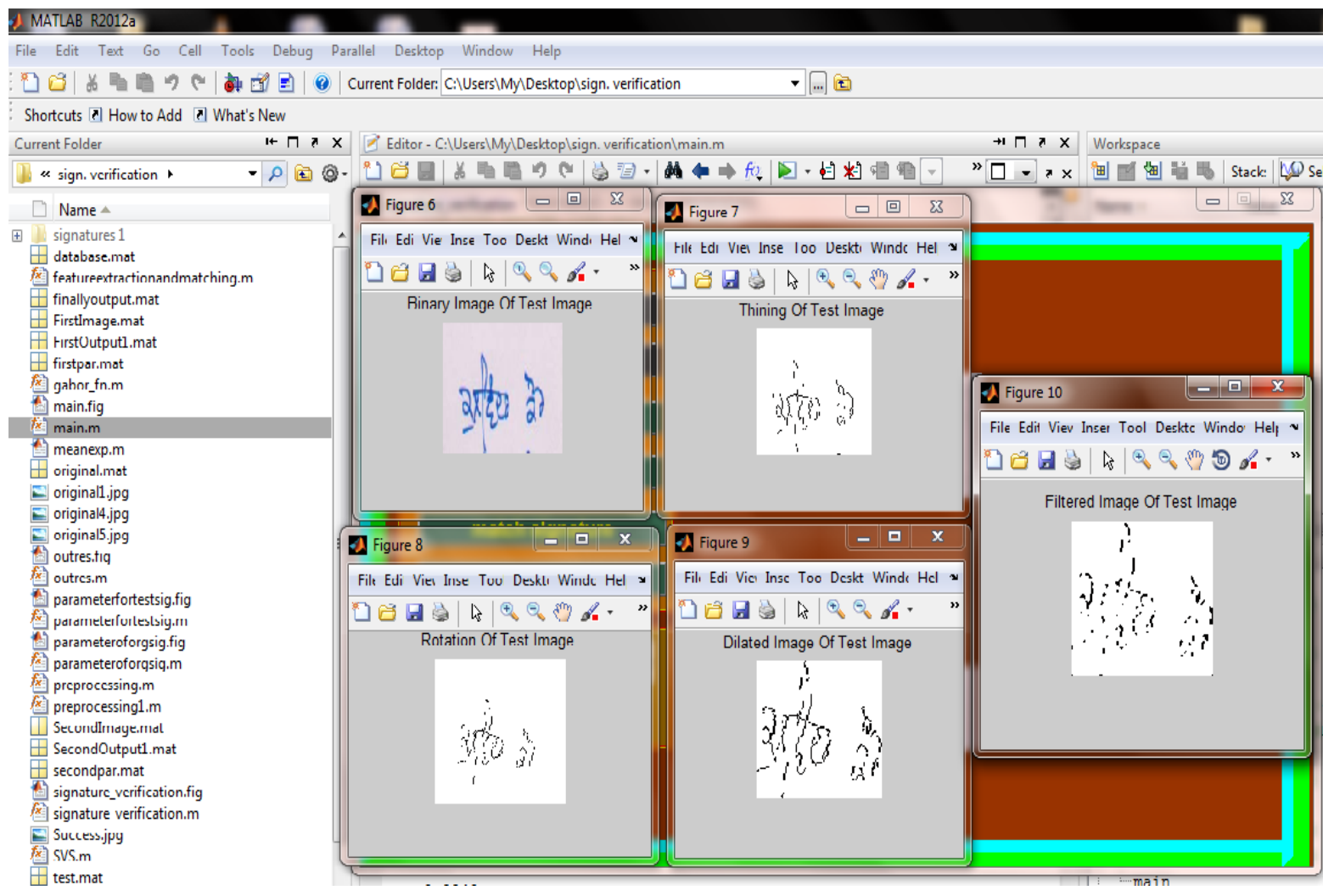

Figure 5: Preprocessing of test image.

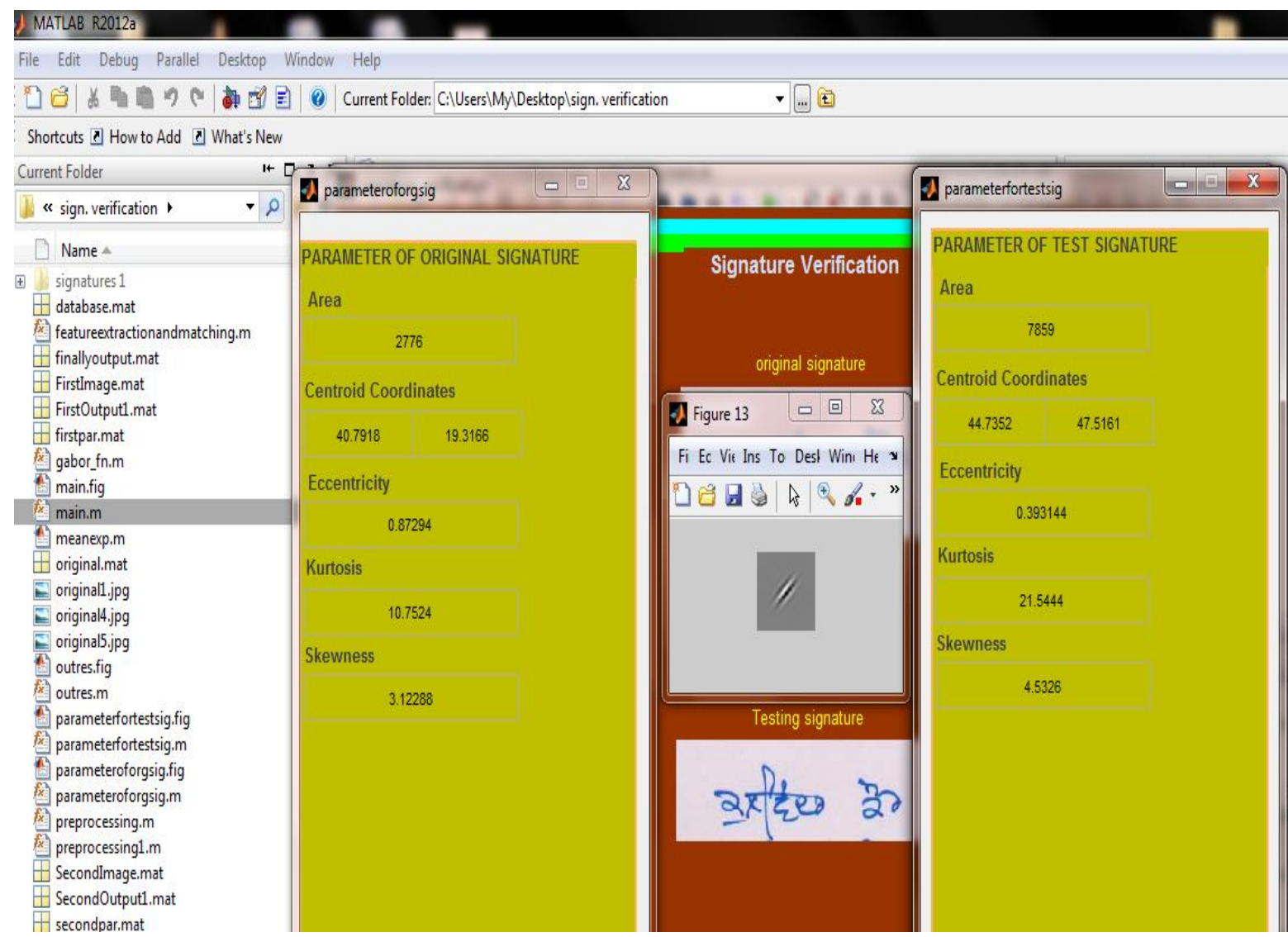

Figure 6: Feature extraction on different parameters using Gabor filter 


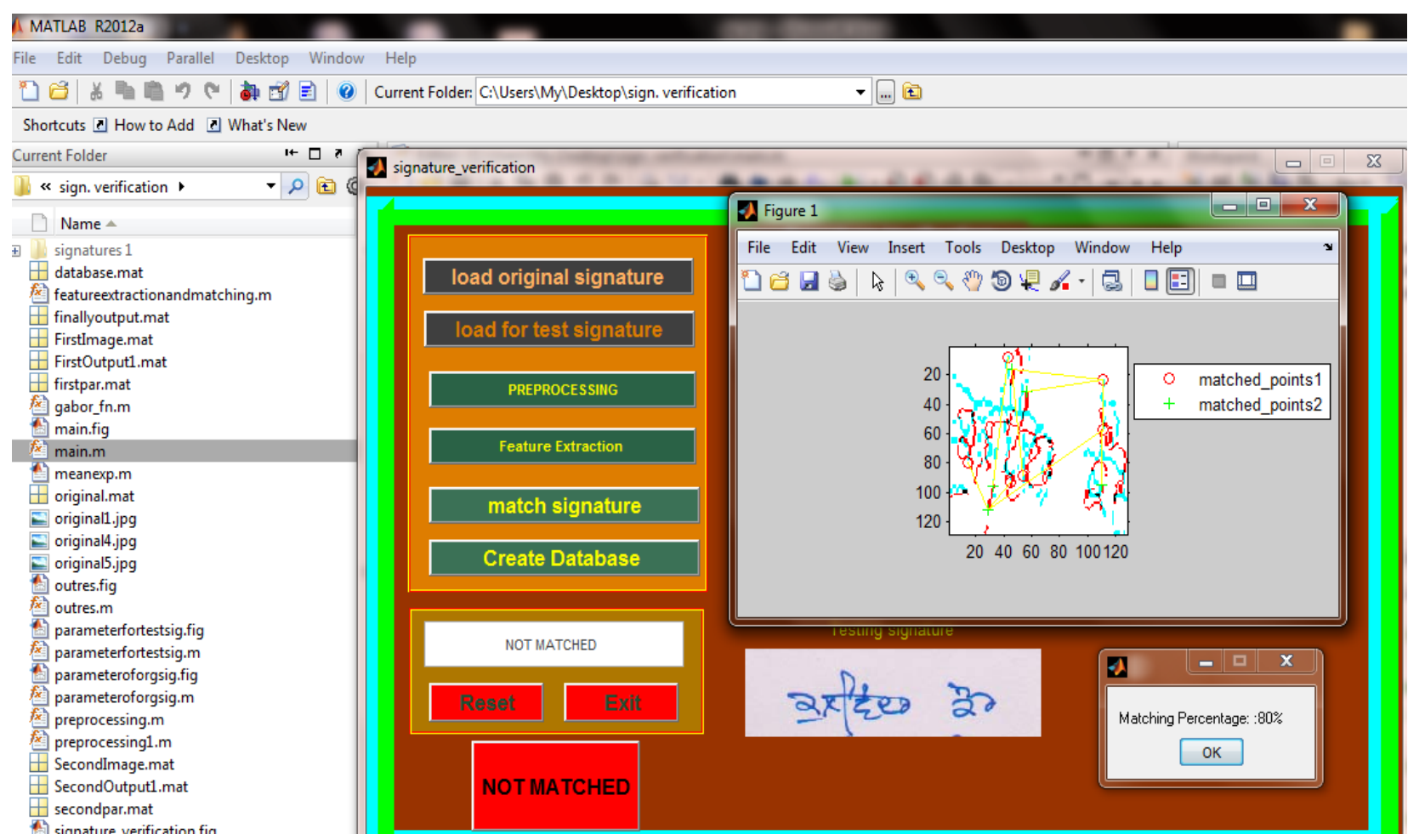

Figure 7: Signature Matching using SURF features and critical point matching

\section{CONCLUSION}

A problem of personal verification and identification is an actively growing area of research. The methods are based on different personal characteristics; movement, lips voice, hand geometry, face, gait, odar, iris, fingerprint and retina are the most commonly used authentication methods. Therefore considerable number of applications is concentrated in the area of electronic commerce and electronic banking systems. Many signature verification techniques have been proposed earlier but they were not secure enough and can be temporarily tampered with so the task was not fulfilled. Signature Verification using Surf feature alone could not provide better results. We use Signature Verification Technique using HMM with surf feature. The results of matching are improved as we use HMM with surf feature technique for matching. Better improved quality of signature and matching results are obtained.

\section{REFERENCES}

[1] Anil K Jain, Arun Ross and Salil Prabhakar, "An Introduction to Biometric Recognition,"IEEE Transactions on Circuits and Systems for Video Technology, vol. 14, no.1, pp. 1-29, 2004.

[2] K.R. Radhika, M.K.Venkatesha and G.N. Sekhar, "OffLine Signature Authentication Based on Moment Invariants Using Support Vector Machine", Journal of Computer Science 6 (3): 305-311, 2010.

[3] Reza Ebrahimpour, Ali Amiri, Masoom Nazari and Alireza Hajiany, "Robust Modelfor Signature Recognition Based on Biological Inspired Features", International Journal of Computer and Electrical Engineering, Vol. 2, No. 4, August, 2010.

[4] A. Piyush Shanker, A.N. Rajagopalan, "Off-line signature verification using DTW", Pattern Recognition Letters 28 (2007) 1407-1414.
[5] Mohammed A. Abdala \& Noor Ayad Yousif, Offline Signature Recognition and Verification Based on Artificial Neural Network, Engineering \& Technology Journal, Vol.27, No.7, 2009.

[6] L. Basavaraj and R.D Sudhakar Samuel, Offline line signature Verification and Recognition: An Approach Based on Four Speeds Stroke Angle, International Journal of Recent Trends in Engineering, Vol 2, No. 3, November 2009.

[7] H. Baltzakis, N. Papamarkos, A new signature verification technique based on a two stage neural network classifier, Engineering Applications of Artificial Intelligence 14 (2001) 95-103.

[8] Jesus F. Vargas, Miguel A. Ferrer, Carlos M. Travieso, Jesus B. Alonso, Offline Signature Verification Based on Pseudo Cepstral Coefficients, 10th International Conference on Document Analysis And Recognition 2009.

[9] Ashwini Pansare, Shalini Bhatia, Handwritten Signature Verification Using Neural Network, International Journal of Applied Information Systems (IJAIS) ISSN: 2249 0868 Foundation of Computer Science FCS, New York, USA Volume 1 No.2, January 2012.

[10] Jean Baptiste Fasquel and Michel Bruynooghe, A hybrid opto- Electronic method for Real time automatic verification of handwritten Signatures, Digital Image Computing Techniques and Applications,21, 22 January 2002, Melbourne, Australia.

[11] Julio Martínez R., Rogelio Alcántara S., Online signature Verification Based on Optimal feature representation and neural Network driven Fuzzy reasoning. 
[12] Prashanth C. R. and K. B. Raja, Offline Signature Verification Based On Angular Features, International Journal of Modeling and Optimization, Vol. 2, No. 4, August 2012.

[13] Shashi Kumar D R, K B Raja, R. K Chattaroy, Sabyasachi Pattanaik, Offline Signature Verification Based on Fusion of Grid and Global Features Using Neural Networks, International Journal of Engineering Science and Technology Vol. 2(12), 2010, 7035-7044.

[14] J.F. Vargas, M.A. Ferrer, C.M. Travieso, and J. B. Alonso. Offline Signature Verification based on pseudocepstral coefficients. 10th IEEE Int Conf on Document Anal. \& Recognition, 2009.

[15] A.I. Al-Shoshan. Handwritten signature verification using image Invariants and Dynamic features. Proc of the IEEE Int Conf on Computer Graphics, Imaging and Visualization (CGIV'06), 2006.

[16] M. Piekarczyk. Hierarchical random graph model for offline handwritten signatures Recognition. IEEE Int Conf on Complex, Intelligent, Software Intensive Systems, 2010.

[17] S.M.S. Ahmad, A. Shakil, M.A. Faudzi, R.M. Anwar. Analysis of 'Goat' within user population of an offline signature biometrics. $10^{\text {th }}$ IEEE Int Conf on Information Science, Signal Processing and their Applications (ISSPA 2010).

[18] J.P. Drouhard, R. Sabourin, and M. Godbout. A neural network Approach to off-line Signature verification using directional PDF. Pattern Recognition, 29(3), (1996), 415--424.

[19] Anil K Jain, Arun Ross and Salil Prabhakar, "An Introduction to Biometric Recognition,"IEEE Transactions on Circuits and Systems For Video Technology, vol. 14, no.1, pp. 1-29, 2004.

[20] Vahid Malekian, Alireza Aghaei, Mahdie Rezaeian and Mahmood Alian, "rapid Offline signature verification base on signature envelope And density partioning" IEEE, 2013.

[21] Ali Karouni, Bassam Daya, samia Bahlak, "offline signature recognition Using neural Network approach" A. Karouni et al. /Procedia Computer Science 3 (2011) 155-161.

[22] Vn Nguyen, Michael Blumenstein Graham Leedham, "global feature For offline Signature verification problems" $10^{\text {th }}$ international Conference on document Analysis and recognition, 2009 